\section{Facharztprüfung zur Erlangung des Facharzttitels FMH für Gastroenterologie}

Aufgrund des Weiterbildungsprogrammes und des Beschlusses des Zentralvorstandes der FMH vom 16. Dezember 1999 ist das Bestehen der Facharztprüfung für diejenigen Kandidatinnen und Kandidaten Voraussetzung für die Erlangung des Facharzttitels FMH für Gastroenterologie, welche ihre Weiterbildung in Gastroenterologie bis am 31. Dezember 2001 nicht abgeschlossen haben. Wer bis Ende 1999 bereits an einer Prüfung teilgenommen hat, muss keine weitere Prüfung absolvieren. Wer die Weiterbildung bis am 31. Dezember 2001 vollständig (einschliesslich Facharztprüfung) abgeschlossen hat, benötigt für die Titelerteilung lediglich eine Teilnahmebestätigung über die absolvierte Facharztprüfung (vgl. Schweizerische Ärztezeitung Nr. 6 vom 7. Februar 2001). Es empfiehlt sich, die Facharztprüfung frühestens im letzten Jahr der reglementarischen Weiterbildung abzulegen (Art. 22 WBO).
Ort: Abteilung für Gastroenterologie, Kantonsspital Luzern

Zeitpunkt: 15. November 2001: schriftlich-theoretische Prüfung (Teil A) und schriftliche Interpretation von Dokumenten bildgebender Verfahren (Teil B)

29. November 2001: mündlich-praktische Prüfung mit Falldiskussion (Teil C)

Prüfungsgebühr: Die FAGAS erhebt eine Prüfungsgebühr von Fr. 600.--

Schriftliche Anmeldung an: PD Dr. med. E. L. Renner, Beauftragter für die Weiter- und Fortbildung FAGAS, Universitätsspital, Abt. Gastroenterologie und Hepatologie, 8091 Zürich, Tel. 0125523 85, Fax 0125545 98, E-mail: eberhard.renner@dim. usz.ch.

Die Anmeldeformulare können bei PD Dr. med. E. L. Renner bezogen werden.

Anmeldefrist: 31 . August 2001

Die Anmeldung gilt mit der Einzahlung der Prüfungsgebühr.

\section{Examen de spécialiste en vue de l'obtention du titre de spécialiste FMH en gastroentérologie}

Se fondant sur le programme de formation postgraduée et la décision du Comité central de la FMH du 16 décembre 1999, la réussite de l'examen de spécialiste est exigée pour le titre de spécialiste FMH en gastroentérologie de tous les candidats n'ayant pas terminé leur formation jusqu'au 31 décembre 1999. Le candidat ayant déjà participé à un examen avant la fin 1999 ne devra pas passer d'autre examen. Le candidat terminant sa formation postgraduée d'ici au 31 décembre 2001 (examen y compris) doit, pour l'obtention du titre, uniquement présenter une attestation de participation à l'examen (cf. BMS n ${ }^{\circ} 6$, du 7 février 2001). Il est recommandé au candidat de passer son examen de spécialiste au plus tôt durant la dernière année de sa formation postgraduée réglementaire (cf. art. 22 RFP).
Lieu: Division de gastroentérologie, Hôpital cantonal Lucerne

Date: 15 novembre 2001: examen théorique écrit (partie A) et interprétation écrite de documents se rapportant à des procédés d'imagerie médicale (partie B)

29 novembre 2001: examen clinique oral avec discussion de cas (partie C)

Taxe d'examen: la SPGES perçoit une taxe de Fr. 600.-.

Inscription par écrit à l'adresse suivante: Dr E. L. Renner, p.-d., Responsable SPGES pour la formation postgraduée, Hôpital universitaire, Division de gastroentérologie et hépatologie, 8091 Zurich, tél. 0125523 85, fax 0125545 98, e-mail: eberhard. renner@dim.usz.ch

Pour les formules d'inscription, veuillez vous adresser au Dr E. L. Renner, p.-d.

Délai d'inscription: 31 août 2001

L'inscription est définitive dès le paiement de la taxe d'examen. 